\title{
Ascitis secundaria a anisakiasis: la importancia de la historia clínica y las pruebas complementarias
}

\author{
Marta Sotelo-García', Mónica Calderón², Juan Crespo ${ }^{3}$, Leticia de las Vecillas ${ }^{4}$, Pablo Garmilla-Ezquerra ${ }^{5}$ \\ ${ }^{1}$ Servicio de Oncología Médica. Hospital Universitario Marqués de Valdecilla. Santander (Cantabria). España \\ ${ }^{2}$ Servicio de Reumatología. Hospital Universitario Marqués de Valdecilla. Santander (Cantabria). España \\ ${ }^{3}$ Servicio de Radiodiagnóstico. Hospital Universitario Marqués de Valdecilla. Santander (Cantabria). España \\ ${ }^{4}$ Servicio de Alergología. Hospital Universitario Marqués de Valdecilla. Santander (Cantabria). España \\ ${ }^{5}$ Servicio de Urgencias. Hospital Universitario Marqués de Valdecilla. Santander (Cantabria). España
}

Recibido: 06/09/2018

Aceptado: 25/10/2018

En línea: 31/12/2018

Citar como: Sotelo-García M, Calderón M, Crespo J, De las Vecillas L, Garmilla-Ezquerra P. Ascitis secundaria a anisakiasis: la importancia de la historia clínica y las pruebas complementarias. Rev Esp Casos Clin Med Intern (RECCMI). 2018 (Dic); 3(3): 118-121. doi: 10.32818/reccmi.a3n3a6.

Autor para correspondencia: Marta Sotelo-García. marta.sotelo@scsalud.es

\section{Palabras clave}

$\triangleright$ Anisakis

$\triangleright$ Ascitis

$\triangleright$ Dolor abdominal

$\triangleright$ Historia clínica

\begin{tabular}{l}
\hline Keywords \\
\hline$\triangleright$ Anisakis \\
$\triangleright$ Ascites \\
$\triangleright$ Abdominal pain \\
$\triangleright$ Medical record \\
\hline
\end{tabular}

\begin{abstract}
Resumen
La anisakiasis es una enfermedad parasitaria provocada por la ingesta de pescados poco cocinados o crudos infestados de Anisakis simplex. Si no se lleva a cabo un correcto procesamiento del alimento para eliminar las larvas de este nemátodo de la familia Anisakidae, éstas permanecen vivas y producen anisakiasis. Las manifestaciones de esta infección son inespecíficas, desde cuadro de dolor abdominal moderado hasta abdomen agudo. Para su orientación diagnóstica, es necesaria una historia clínica detallada y pruebas de imagen complementarias pertinentes que sugieran y apoyen el diagnóstico.

Abstract
Anisakiasis is a parasitic disease caused by eating undercooked or uncooked fish in which correct processingis
not carried out for the elimination or death of Anisakis simplex (AS) larvae. AS is a nematode of the Anisakiadae
family. Symptoms are very unspecific, from moderate abdominal pain to an acute pain that can suggest need for
surgery. For its diagnostic orientation, a detailed clinical history and relevant complementary imaging tests that
suggest and support the diagnosis are necessary.
\end{abstract}

\section{Puntos destacados}

$\triangleright$ La infestación por Anisakis es cada vez más frecuente, y es necesario tener en mente esta causa de dolor abdominal para elaborar un diagnóstico diferencial completo, o para orientar las pruebas diagnósticas.

$\triangleright$ Se presenta este caso a raíz de un caso atendido en nuestro Servicio de Urgencias.

\section{Introducción}

El dolor abdominal es uno de los motivos de consulta más frecuentes en los Servicios de Urgencias. Posee un amplio diagnóstico diferencial, por lo que es importante una buena historia clínica y exploración física, acompañada de ecografía clínica para, posteriormente, realizar estudios más dirigidos y obtener una mayor seguridad diagnóstica ${ }^{1-4}$.

Presentamos el caso de un paciente con dolor abdominal y ascitis en el que la historia clínica y las pruebas diagnósticas nos hicieron sospechar el diagnós- tico de anisakiasis, pudiendo confirmarse en estudios inmunológicos a posteriori. La anisakiasis es una infección parasitaria que puede cursar con dolor abdominal y reacciones alérgicas en relación con la ingesta previa de pescado crudo o poco cocinado ${ }^{1,2}$. Es una causa poco frecuente de dolor abdominal en Urgencias, pero puede provocar cuadros de ascitis o suboclusivos, por lo que es fundamental la sospecha clínica inicial para su diagnóstico y tratamiento definitivo sin generar yatrogenia ${ }^{3-5}$.

\section{Caso clínico}

Presentamos el caso de un paciente varón de 35 años, sin antecedentes personales de interés, que consulta de madrugada en el Servicio de Urgencias por dolor abdominal de 3 días de evolución, controlado parcialmente con analgesia, pero que esa misma noche se intensifica, con hiporexia y vómitos. No presentaba fiebre ni alteración del hábito intestinal. En la exploración física inicial estaba afebril y hemodinámicamente estable, con dolor intenso a la palpación en epigastrio, sin signos de irritación peritoneal. El hemograma 
Sotelo-García M, Calderón M, Crespo J, De las Vecillas L, Garmilla-Ezquerra P. Ascitis secundaria a anisakiasis: la importancia de la historia clínica y las pruebas complementarias

mostró 20.100 leucocitos/ $\mu$ l (segmentados 88,0\%, linfocitos 8,0\%, monocitos $4,0 \%$, eosinófilos $0 \%$, basófilos $0 \%$ ), sin otros hallazgos analíticos relevantes. Clínicamente, el paciente mejoró con la administración de $2 \mathrm{~g}$ de metamizol intravenoso. Al cambio de guardia, el paciente estaba asintomático. Ante la ausencia de un diagnóstico claro, se realizó una ecografía a pie de cama, evidenciando la presencia de moderada cantidad de líquido libre intraabdominal (Figura 1), por lo que se solicitó al Servicio de Radiodiagnóstico una tomografía axial computarizada (TAC) abdominal, prueba en la que se describió engrosamiento difuso de las paredes de la cámara gástrica edema de la grasa circundante y líquido libre periesplénico y perihepático (Figura 2).

Ante la impresión radiológica de una gastritis por infestación por Anisa kis, se realizó una nueva anamnesis al paciente, que confirmó la ingesta de boquerones en vinagre crudos los días previos y esa misma noche. Posteriormente, se realizó una gastroscopia que objetivó una mucosa congestiva con engrosamiento de los pliegues en cuerpo gástrico, sin visualización del parásito. Se tomaron muestras de biopsia, que posteriormente describieron una gastritis crónica superficial sin eosinofilia.

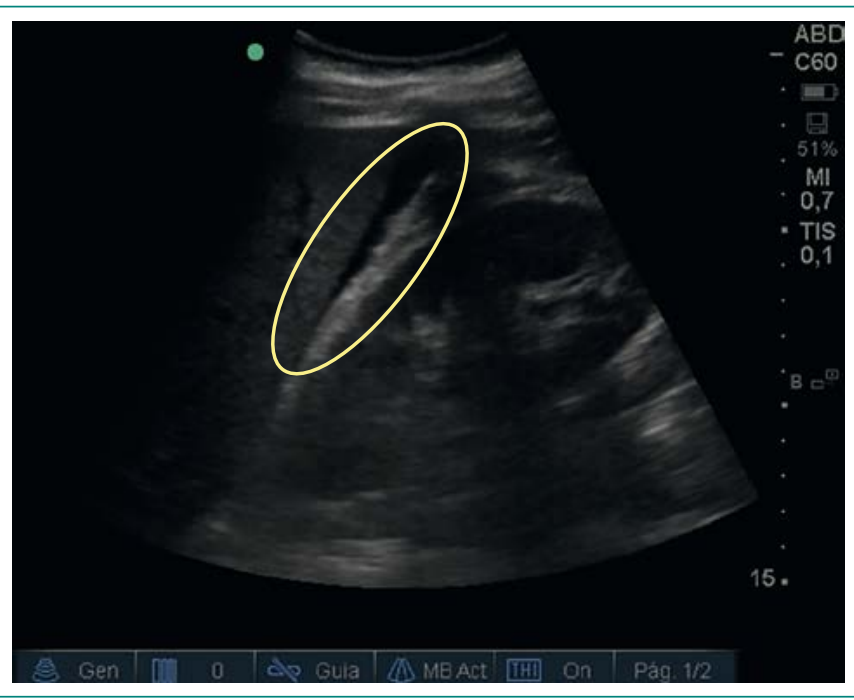

Figura 1. Ecografía clínica: líquido libre en Morrison (circulo amarillo)
Además, se solicitó un estudio inmunohistoquímico para determinar los valores de la IgE específica frente a Anisakis simplex en suero (ImmunoCAP) con valor inicial de 30,3 kU $/$ I (valor positivo $>0,35 \mathrm{kU} / \mathrm{A}$ ). El paciente fue dado de alta a las 10 horas de la valoración inicial, asintomático y sin complicaciones posteriores.

El estudio inmunoalérgico se completó un mes más tarde en consultas de Alergología, donde se realizó prueba cutánea con extracto completo de Anisakis simplex (Bial Aristegui, $10 \mu \mathrm{g} / \mathrm{ml}$ ), presentando una pápula de $10 \times 10 \mathrm{~mm}$ de diámetro (resultado positivo $>3 \mathrm{~mm}$ ) y determinando un nuevo valor de lgE específica, que se había elevado a 524 kU $\mathrm{A} /$.

\section{Discusión}

La anisakiasis es una enfermedad causada por alimentos contaminados con larvas vivas del parásito Anisakis simplex, nemátodo de la familia Anisakidae ${ }^{2,6}$. Existen varias formas según la presentación clínica y el mecanismo fisiopatológico:

- Anisakiasis gastrointestinal, con infestación exclusiva del tracto digestivo, pudiendo generarse cuadros asintomáticos cuando el parásito tiene presencia luminal o no invasiva y las larvas son eliminadas mediante vómitos o en las heces; o sintomáticos, cuando hay invasión gástrica o intestinal, caracterizada por dolor abdominal. Los síntomas pueden aparecer entre las primeras 5-12 horas y hasta varios días tras la exposición al parásito, lo que dificulta el diagnóstico.

- Anisakiasis alérgica. Cuadros de hipersensibilidad lgE mediada con clínica fundamentalmente cutánea de urticaria y angioedema, pudiendo evolucionar a anafilaxia, sin aparente parasitación.

- Anisakiasis gastroalérgica. Cuando ambos cuadros coexisten en un mismo paciente.

La presentación clínica se suele caracterizar por un cuadro inespecífico de febrícula y dolor abdominal, tipo subagudo, con náuseas y vómitos. Si la infestación es gástrica, en raras ocasiones se puede presentar como una hemorragia digestiva alta secundaria a ulceración de la mucosa gástrica; $y$, si es intestinal, como un cuadro de abdomen agudo (con líquido libre, obstrucción intestinal... $)^{1,3,4}$. La ingesta de pescado crudo o la visualización del parásito en el pescado poco cocinado (escabeche, salazones,
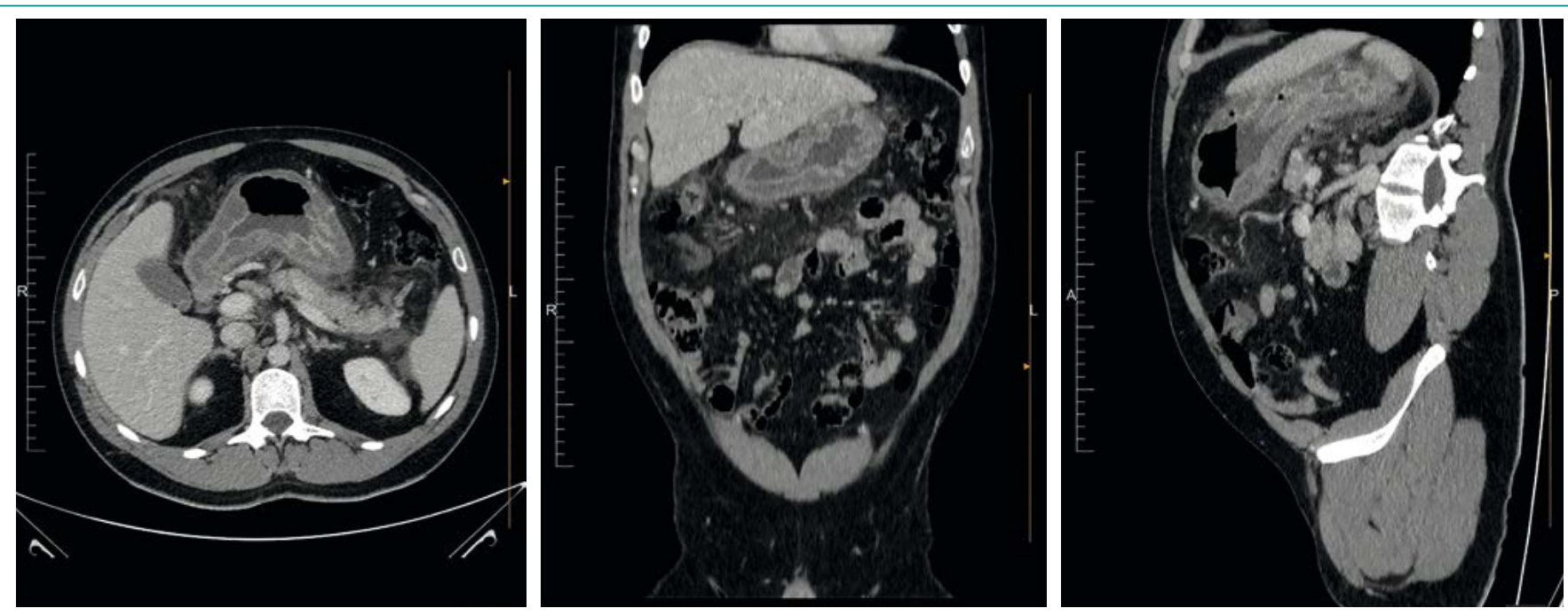

Figura 2. TAC abdominal: engrosamiento difuso de las paredes de la cámara gástrica, edema de la grasa circundante y líquido libre periesplénico y perihepático 

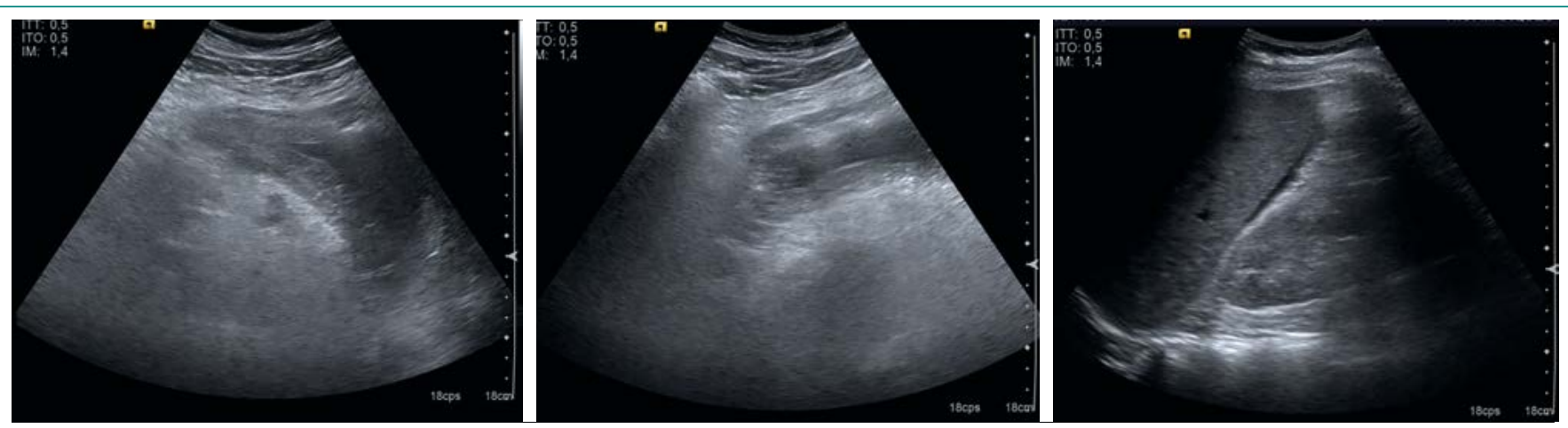

Figura 3. Ecografía abdominal: engrosamiento parietal gástrico

en vinagre o ahumados) son las pistas que pueden orientar la sospecha diagnóstica ${ }^{2,3}$.

En ocasiones, la afectación intestinal que cursa con cuadro de abdomen agudo y la ausencia de un diagnóstico claro conducen a una intervención quirúrgica urgente (laparoscopia, laparotomía exploradora e incluso resección de la porción afectada de asas intestinales). Por lo que la sospecha clínica es fundamental para evitar yatrogenia. En los casos descritos en la literatura al igual que el nuestro, el cuadro se resolvió con dieta absoluta y tratamiento sintomático ${ }^{3,4}$.

Las técnicas de imagen pueden mostrar un hipoperistaltismo ecográfico, estenosis luminal, un segmento intestinal con edema de pared y dilatación proximal de asas de intestino, sin una clara causa obstructiva, ascitis o líquido libre $^{3,5}$.

En los casos de sospecha diagnóstica de anisakiasis en el momento precoz existe indicación de realizar endoscopia digestiva alta ${ }^{4,5}$. La visualización del parásito y la extracción del mismo en la fase aguda nos ayudan en el diagnóstico y tratamiento definitivo ${ }^{4-6}$. Es posible también que, si nos demoramos en esta prueba, ya no sea factible la captura del mismo, y como único hallazgo se encuentre una mucosa edematosa alrededor del punto de invasión de la pared, preferentemente siguiendo la curvatura mayor del estómago. Este detalle es importante porque la congestión de la mucosa puede dificultar el estudio y es necesario conocer las localizaciones más frecuentes de Anisakis ${ }^{4,5}$.

En el estudio anatomopatológico, puede encontrarse edema con abundante infiltrado eosinófilo o, en un momento más avanzado, granulomas epitelioides con células gigantes de cuerpo extraño $0^{3,4}$.

Para determinar si el paciente se ha sensibilizado al parásito, incluso aunque no haya presentado clínica sugestiva de reacción alérgica en un primer episodio, es necesario un estudio inmunológico. Mediante la determinación de lgE específica frente a Anisakis simplex por InmunoCAP o ELISA y un estudio in vivo con pruebas cutáneas se determinará el riesgo del paciente a sufrir cuadros alérgicos o gastroalérgicos en futuros contactos con el parásito ${ }^{2,7}$. La presencia en sangre periférica de eosinofilia tiene escaso valor diagnóstico, ya que inicialmente, en la mayoría de los casos, se presentará únicamente leucocitosis, debido a que en los primeros días los eosinófilos son reclutados en los tejidos inflamados y suelen ser necesarios más de 10 días de evolución del cuadro para observar su aumento en sangre periférica ${ }^{1,4-6}$. La presencia de estas células en el líquido ascítico es muy sugestiva de infección ${ }^{4,5}$. Algunos criterios útiles para el diagnóstico podrían ser los ya descritos (Tabla 1). Además, podemos encontrar manifestaciones de tipo extraintestinal caracterizadas por síntomas alérgicos, y es que el 30\% de los enfermos sensibilizados sufrirán cuadros de urticaria o angioedema tras la toma de pescado crudo $^{6,8}$.

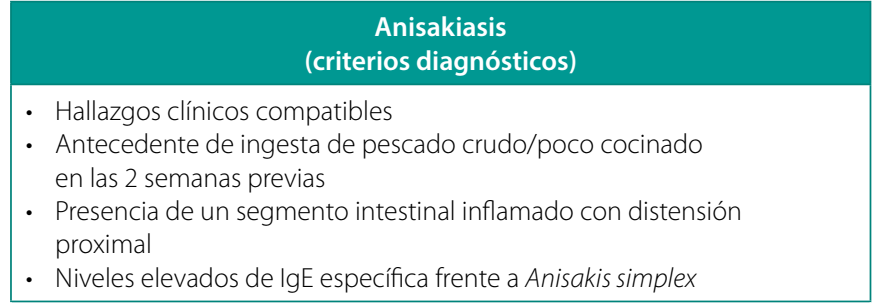

Tabla 1. Criterios diagnósticos de anisakiasis

\section{Conclusiones}

Como ideas fundamentales en la valoración del dolor abdominal, podemos concluir que la historia clínica es imprescindible para una buena orientación diagnóstica en pacientes con ingesta de pescado crudo o poco cocinado en días previos, junto con la ecografía a pie de cama, que nos ayudará a orientar mejor los estudios complementarios ${ }^{1,2,6}$. Debemos incluir la anisakiasis en el diagnóstico diferencial del abdomen agudo con líquido libre intraabdominal y ausencia de otros hallazgos en las pruebas de imagen ${ }^{5}$.

Todo ello es fundamental a la hora del diagnóstico de este tipo de pacientes, ya que podría tener importante trascendencia en el manejo terapéutico, asociando un ingreso hospitalario para realizar otros estudios $u$ otras acciones que pudiesen implicar yatrogenia o aumento de los costes sanitarios ${ }^{3}$.

Además, las recomendaciones dietéticas específicas al paciente serían claves en la prevención de nuevos cuadros de anisakiasis, que pueden ser más severos que el inicial ${ }^{2,5-7}$.

\section{Bibliografía}

1. López Peñas D, Ramírez Ortiz LM, Del Rosal Palomeque R, López Rubio F, Fernández-Crehuet Navajas R, Miño Fugarolas G. Anisakiasis en España: una enfermedad creciente. Revisión. Gastroenterol Hepatol. 2000; 23: 307-311.

2. Daschner A, Cuéllar C, Rodero M. The Anisakis allergy debate: does an evolutionary approach help? Trends Parasitol. 2012 Jan; 28(1): 9-15. doi: 10.1016/j.pt.2011.10.001.

3. Shrestha S, Kisino A, Watanabe M, Itsukaichi H, Hamasuna K, Ohno G, et al. Intestinal anisakiasis treated successfully with conservative therapy: importance of clinical diagnosis. World J Gastroenterol. 2014 Jan 14; 20(2): 598602. doi: 10.3748/wjg.v20.i2.598.

4. Repiso Ortega A, Alcántara Torres M, González de Frutos C, De Artaza Varasa T, Rodríguez Merlo R, Valle Muñoz J, et al. Anisakiasis gastrointestinal. 
Sotelo-García M, Calderón M, Crespo J, De las Vecillas L, Garmilla-Ezquerra P. Ascitis secundaria a anisakiasis: la importancia de la historia clínica y las pruebas complementarias

Estudio de una serie de 25 pacientes. Gastroenterol Hepatol. 2003 Jun-Jul; 26(6): 341-346.

5. Chung YB, Lee J. Clinical characteristics of gastroallergic anisakiasis and diagnostic implications of immunologic tests. Allergy, Asthma Immunol Res. 2014 May; 6(3): 228-233. doi: 10.4168/aair.2014.6.3.228

6. Valiñas B, Lorenzo S, Eiras A, Figueiras A, Sanmartín ML, Ubeira FM. Prevalence of and risk factors for IgE sensitization to Anisakis simplex in a Spanish population. Allergy. 2001; 56: 667-671.
7. Cuéllar C, Daschner A, Valls A, De Frutos C, Fernández-Fígares V, Anadón $A M$, et al. Ani s 1 and Ani s 7 recombinant allergens are able to differentiate distinct Anisakis simplex-associated allergic clinical disorders. Arch Dermatol Res. 2012 May; 304(4): 283-288. doi: 10.1007/s00403-0121206-8.

8. Ventura MT, Napolitano S, Menga R, Cecere R, Asero R. Anisakis simplex hypersensitivity is associated with chronic urticaria in endemic areas. Int Arch Allergy Immunol. 2013; 160(3): 297-300. doi: 10.1159/000339869. 